\title{
Thermodynamics of Formation of Porous Polymeric Membrane from Solutions
}

\author{
Shigenobu MATSUDA \\ Fundamental Research Laboratory of Fibers and Fiber-Forming Polymers, \\ Asahi Chemical Industry Co., Ltd., \\ 11-7 Hacchonawate, Takatsuki, Osaka 569, Japan
}

(Received November 26, 1990)

\begin{abstract}
In order to give better and quantitative understanding of the pore forming mechanism in the solvent casting method, a theory was presented as an example of the application of phase equilibrium. Mechanism of formation of critical nucleus from homogeneous polymer solution in the metastable region of the phase diagram was investigated in accordance with the activation energy of nucleation. The growth rate of nucleus by diffusion to the primary particle and the time of attainment of phase equilibria of whole system were determined under the assumption of local equilibrium between nucleus and sorrounding sphere. Formation of secondary particle by amalgamation was tried to explain by use of the particle Monte-Carlo simulation approach.

KEY WORDS Solvent Casting Method / Thermodynamics

Critical Nucleus / Phase Diagram / Activation Energy / Diffusion / Primary Particle / Secondary Particle / MonteCarlo simulation /
\end{abstract}

Among numerous methods proposed hitherto for preparing polymeric membrane, the solvent cast method is a method of vast technological importance, as it enables us to produce membrane with a wide range of mean pore sizes and phase separation plays an important role in the formation of the membrane. In this article, more generalized theory as an extension of pioneering work of Kamide and Manabe ${ }^{1}$ was presented especially on the nucleation and the growth of nucleus to primary particle. Summary of these works has been published in the book entitled "Thermodynamics of Polymer Solutions" by Kamide. ${ }^{2}$

The underlying mechanism of pore formation in the solvent casting method is schematically demonstrated in Figure 1. The original form of this figure was first presented by Kamide and Manabe ${ }^{2}$ in 1985. Depending on the initial polymer concentration $\mathbf{v}_{\mathrm{p}}{ }^{\mathrm{o}}$, either the polymer-rich phase or the polymer-lean phase separates as the disperse phase from the solution. If initial polymer concentration is less than the critical solution concentration $v_{p}{ }^{c}$, the polymer-rich phase separates as small particles suspended in a medium, which is polymer-lean phase, and these particles grow by amalgamation. The interstitial space between particles gives non-circular pore.

When $\mathbf{v}_{\mathbf{p}}{ }^{\mathrm{o}}$ is larger than $\mathrm{v}_{\mathrm{p}}{ }^{\mathrm{c}}$, the polymer-lean phase is separated as shown in Figure $1 b^{\prime}$ to $k^{\prime}$. The aggregated polymer-lean phase particles are themselves circular, smooth pores. Even in this case, stage $g^{\prime}$ should be stable in order to prepare the microporous membrane. These steps strongly suggests that circular pores will be found from concentrated solution. More quantitative explanation will be presented 


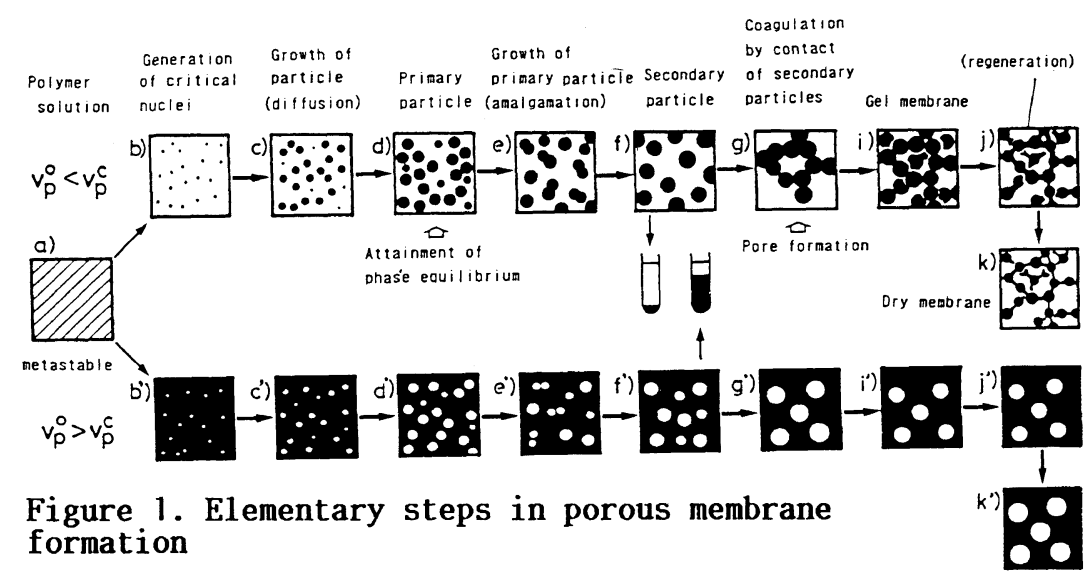

in this article, especially on generation of critical nuclei and growth of particle to primary particle. In short, the pore formation model is based on the "particle-growth concept", which has its basis on the observation of Kamide and Manabe.

\section{THEORETICAL BACKGROUND}

The generation and growth of polymer particles by phase separation from homogeneous solution is based principally on the same concepts used to explain the condensation of liquid droplets from supper saturated vapours or the formation of ice particles from super-cooled liquids. Figure 2 shows the cloud point curve (full line), spinodal curve (broken line) and critical solution point (open circle) for monodisperse polymer ( $\mathrm{X}$-mer; $\mathrm{X}$, molar volume ratio of polymer to solvent) / single solvent system and Figure 3 shows the Gibbs free energy change $\Delta G_{v}$ per unit volume at a given temperature $\mathrm{T}_{\mathrm{P}}$ for $\mathrm{X}$-mer / single solvent system. $v_{x_{(1)}}$ and $v_{x(2)}$ are the polymer volume fractions of the polymer-lean and -rich phases in equilibrium and $\mathbf{V}_{\mathbf{X}_{(1)}}{ }_{\mathrm{SP}}$ and $\mathrm{V}_{\mathrm{X}_{(2)}}{ }_{\mathrm{SP}}$ are spinodal concentrations. In these figures, points $A$ and $B$ are two-phase equilibrium points and points $C$ and $D$ are spinodal points. If the initial polymer concentration $v_{x}{ }^{0}$ lies at temperature $\left(T_{P}\right)$ between $A$ and $B$, the two-phase separation occurs. When initial polymer concentration lies between $A$ and $C$ or between $D$ and $B$,

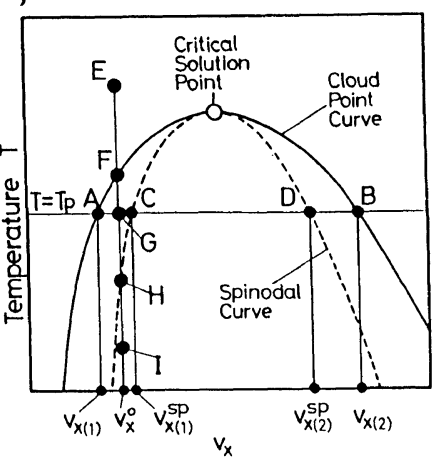

Figure 2. Phase diagram of $\mathrm{X}$-mer / solvent system.

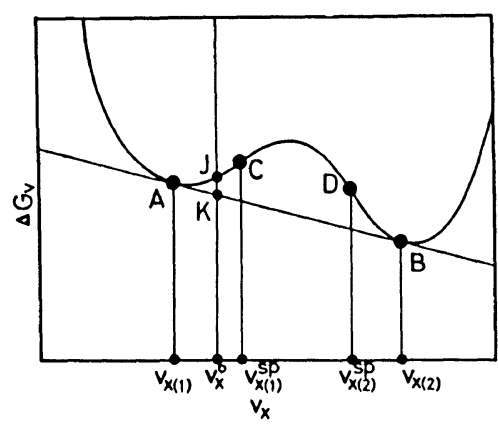

Figure 3. Gibbs free energy of unit volume. the polymer solution can exist as a metastable single phase, from which two-phase separation is initiated by formation of nuclei. In other words, in homogeneous polymer solutions in the metastable region of the phase diagram between cloud point curve and spinodal curve, for example, at point $G$ in Figure 2, or point $J$ in Figure 3, critical nuclei are formed by "concentration fluctuation" and can grow further in size spontaneously. The generation of the precipitated nuclei is always time retarded and the nuclei thus formed grow by passing through a potential barrier. On the other hand, under the appropriate conditions the 
precipitation occurs by passing through the critical point is instantaneous because of lack in potential barrier. This is spinodal decomposition. In this article, the pore formation mechanism is explained from the standpoint of nucleation.

Phase equilibrium of polymer / solvent system

Before starting an explanation of nucleation, the phase equilibria of polymer: / single solvent system will be presented. First, the spinodal curve, the binodal curve, and the critical solution point of polymer solutions will be briefly explained for X-mer / single solvent system.

The mean molar Gibbs free energy of mixing of the system, $\Delta G$, is schematically plotted in Figure 4 as a function of the polymer concentration and temperature $T$ at constant pressure $P$. In the figure the line connecting the points of inflection is the spinodal curve. In other words, this is the line at which the second differential of free energy becomes zero. The line connecting the point of contact of the double tangent is the binodal curve. The line connecting the energy of mixing points at

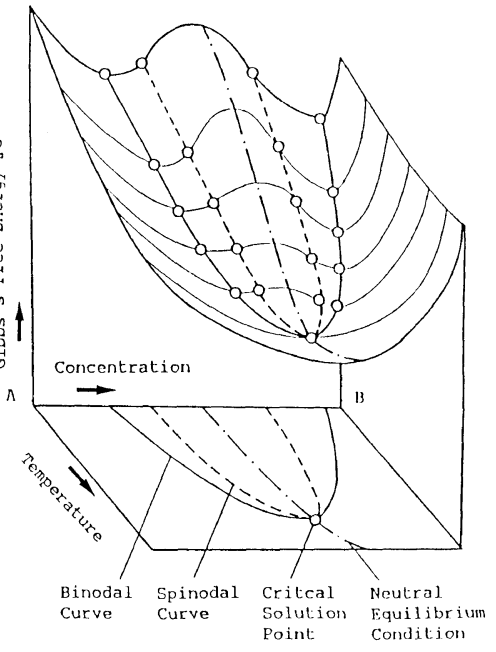

Figure 4. Gibbs free energy of mixing. which the third differential of free energy in concave plane between two spinodal branches, is often described as the neutral equilibrium condition. The point at which the binodal curve coincides with the spinodal curve, is the critical point of the solution at a given pressure. At the critical solution point, free energy is a minimum and of course the neutral equilibrium condition is satisfied. The critical point can be determined as the point at which the spinodal condition and the neutral equilibrium condition are concurrently satisfied.

Thermodynamic interaction parameter between polymer and solvent $\chi$, is given by eq 1 phenomenologically as a power series of the concentration. ${ }^{3-6}$

$\chi=\chi_{0}\left(1+\sum_{j=1}^{n} p_{j} v_{p}^{j}\right)$.

Here, $v_{p}$ is overall polymer concentration, $p_{j}(j=1, \cdots, n)$, concentration dependence parameters and $\chi_{0}$ is divided into two part, temperature dependent part and molecular weight dependent part, as eq 2,

$$
\begin{aligned}
\chi_{0} & =\chi_{\text {oo }}\left(1+\frac{\mathbf{k}^{\prime}}{\mathrm{X}_{\mathrm{n}}}\right) \\
& =\left\{\left(\frac{1}{2}-\psi_{\mathrm{o}}\right)+\frac{\psi_{\mathrm{o}} \theta}{\mathrm{T}}\right\}\left\{1+\frac{\mathbf{k}_{\mathbf{0}}}{\mathrm{X}_{\mathrm{n}}}\left(1-\frac{\theta}{\mathrm{T}}\right)\right\},
\end{aligned}
$$

where $\mathbf{k}^{\prime}$ (or $\mathrm{k}_{0}$ ) is the molecular weight dependence parameter, $\theta$, Flory temperature, $\psi_{0}$, entropy parameter and $\mathrm{X}_{\mathrm{n}}$, the number average degree of polymerization. Chemical potentials of the solvent $\Delta \mu_{0}$ and $X_{1}-$ mer $\Delta \mu_{x_{i}}$ of the multicomponent polymer / single solvent system are given by these equations, respectively. ${ }^{3-6}$

$$
\begin{aligned}
\Delta \mu_{0}=\tilde{R} & \operatorname{RT}\left\{\ln \left(1-v_{p}\right)+\left(1-\frac{1}{X_{n}}\right) v_{p}+\chi v_{p}{ }^{2}\right\} \\
\Delta \mu_{x_{i}}= & \tilde{R T}\left[\ln v_{x_{i}}-\left(X_{i}-1\right)+X_{i}\left(1-\frac{1}{X_{n}}\right) v_{p}\right. \\
& +X_{i}\left(1-v_{p}\right)^{2} \chi_{o o}\left[\left(1+\frac{k^{\prime}}{X_{n}}\right)\left\{1+\sum_{j=1} \frac{p_{j}}{j+1}\left\{\sum_{q=0}^{j}(q+1) v_{p}{ }^{q}\right\}\right\}\right. \\
& \left.\left.+k^{\prime}\left(\frac{1}{X_{i}}-\frac{1}{X_{n}}\right)\left\{\frac{1}{1-v_{p}}+\sum_{j=1}^{n} \frac{p_{j}}{j+1}\left(\sum_{q=0} \frac{v_{p}^{q}}{1-v_{p}}\right)\right\}\right]\right] \quad(i=1, \ldots, m) .
\end{aligned}
$$


Here, $m$ is the total number of different molar mass components in the polymer sample, $\mathbb{R}$, gas constant. $m$ is always order of from $10^{2}$ to $10^{4}$.

Spinodal curve of multicomponent polymer / single solvent system is given by this determinant, spinodal condition, which is the second variable of the Gibbs free energy: ${ }^{3}$

$\left|\Delta \mathrm{G}^{\prime}\right|=\left|\begin{array}{cccc}\Delta \mathrm{G}_{11} & \Delta \mathrm{G}_{12} & \cdots & \Delta \mathrm{G}_{1 \mathrm{~m}} \\ \Delta \mathrm{G}_{21} & \Delta \mathrm{G}_{22} & \cdots & \Delta \mathrm{G}_{2 \mathrm{~m}} \\ \vdots & \vdots & & \vdots \\ \Delta \mathrm{G}_{\mathrm{m} 1} & \Delta \mathrm{G}_{\mathrm{m} 2} & \cdots & \Delta \mathrm{G}_{\mathrm{mm}}\end{array}\right|=0$.

Critical solution point is determined by solving the simultaneous equation of spinodal condition and neutral equilibrium condition. Neutral equilibrium condition is the third variable of the Gibbs free energy and is given by eq $6:^{3}$

$\left|\Delta G^{\prime} \cdot\right|=\left|\begin{array}{cccc}\frac{\partial\left|\Delta G^{\prime}\right|}{\partial v_{X_{1}}} & \frac{\partial\left|\Delta G^{\prime}\right|}{\partial v_{X_{2}}} & \cdots & \frac{\partial\left|\Delta G^{\prime}\right|}{\partial v_{\mathrm{Xm}_{m}}} \\ \Delta G_{21} & \Delta G_{22} & \cdots & \Delta G_{2 m} \\ \vdots & \vdots & \cdots & \vdots \\ \Delta G_{m 1} & \Delta G_{m 2} & \cdots & \Delta G_{m m}\end{array}\right|=0$.

Here, $\Delta G_{i, j}$ is the second order differential of the Gibbs free energy change of mixing per unit volume $\Delta G_{v}$ with respect to the volume fractions of $X_{i}-$ mer and $X_{j}-$ mer, $v_{X_{i}}$ and $v_{X_{j}} \cdot \Delta G_{v}$ and $\Delta G_{i j}$ are expressed by following equations, respectively. ${ }^{3}$

$\Delta G_{v}=v_{0}\left(\frac{\Delta \mu_{0}}{V_{0}}\right)+\sum_{i=1}^{m} v_{X_{i}}\left(\frac{\Delta \mu_{X_{i}}}{X_{i} v_{0}}\right)$

$\Delta G_{i j}=\left(\frac{\partial^{2} \Delta G_{v}}{\partial v_{X i 1} \partial v_{X j}}\right) T, P, v_{x k}$

$(\mathbf{i}, \mathbf{j}=1, \cdots, m ; k \neq i, j)$.

Here, $V_{0}$ is the molar volume of the solvent which is assumed to coincide with that of repeating unit of the polymer. By substituting the chemical potentials (eqs 3 and 4 ) into eqs 5-8, the following equations are finally obtained as spinodal and neutral equilibrium conditions, respectively. ${ }^{3}$

$\frac{1}{X_{w} v_{p}}+\frac{1}{1-v_{p}}-\chi_{00}\left\{1+k^{\prime}\left(1+\frac{1}{X_{n}}-\frac{X_{w}}{X_{n}}\right)\right\}\left\{2+\sum_{j=1}^{n} p_{j}(j+2) v_{p}{ }^{j}\right\}=0$,
$\frac{1}{\left(1-v_{p}\right)^{2}}-\frac{X_{z}}{\left(X_{w} v_{p}\right)^{2}}-\chi_{o 0}\left\{1+k^{\prime}\left(1+\frac{1}{X_{n}}-\frac{X_{w}}{X_{n}}\right)\right\}\left\{\sum_{j=1}^{n} p_{j} j(j+2) v_{p}^{j-1}\right\}=0$.

When polymer is strictly monodisperse $X$-mer and molecular weight dependence of $\chi$-parameter is neglected, both equations easily reduces to these equations, respectively,

$\frac{1}{1-v_{x}}+\frac{1}{X v_{x}}-\chi_{0}\left(2+3 p_{1} v_{x}+4 p_{2} v_{x}{ }^{2}\right)=0$,

$\frac{1}{\left(1-v_{X}\right)^{2}}-\frac{1}{X v_{x}^{2}}-\chi_{0}\left(3 p_{1}+8 p_{2} v_{p}\right)=0$,

where 1st and 2nd order concentration dependence of $\chi$ are considered. $\chi_{0}$ is converted into temperature through use of this relation, $\mathrm{T}=\frac{\psi_{0} \theta}{\chi_{0}+\psi_{0}-0.5}$

The method of determining the cloud point curve of multicomponent polymer / single solvent system have already established in 1984 on the basis of the Gibbs two-phase equilibrium conditions, ${ }^{3,6}$

$\Delta \mu_{0}(1)=\Delta \mu_{0}(2)$,

$\Delta \mu \times x_{(1)}=\Delta \mu \times x_{(2)}, \quad(i=1, \cdots, m)$.

Figure 5 represents some example of calculated cloud point curve under the condition of $\mathrm{p}_{1}=0.6, \mathrm{p}_{2}=0$ and $\mathrm{k}_{0}=0 .{ }^{3}$ Molecular weight distribution is assumed to follow the Schulz-Zimm type and polymolecularity is changed from 1 to 4. Regardless of the polymolecularity 
of the polymer, cloud point curve is highly unsymmetrical with respect to the polymer concentration. This dissymmetry in cloud point curve is well known experimentally and can be theoretically explained in terms of its large molar volume ratio $X_{i}$ compared to the solvent.

For the strictly monodisperse polymer solution the cloud point curve should be in excellent agreement

with binodal curve, in other

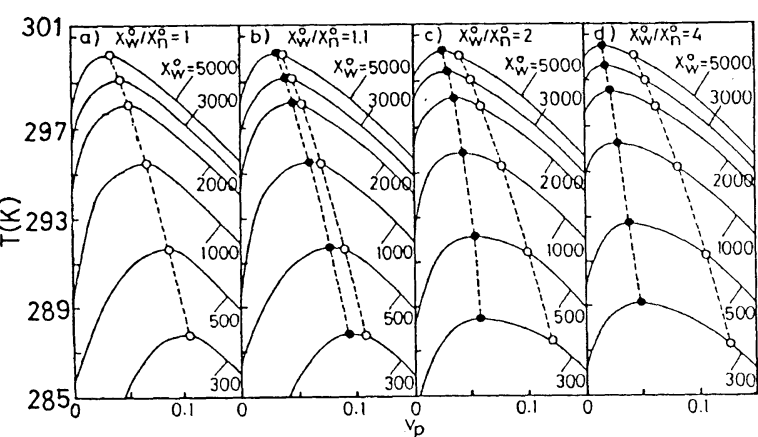

Figure 5. Cloud point curve of multicomponent polymer / solvent system. words, coexistence curve. The critical point, independently calculated by a different procedure from cloud point curve as described and denoted by open circle, lies just on the cloud point curve which verifies the reliability of the calculation. For the monodisperse polymer, the critical point coincides very well with the top of the cloud point curve, so called threshould point denoted by closed circle in these figures. As the polydispersity of the polymer increases, critical points shift to the higher $v_{p}$ region causing them differ from the threshold point significantly. ${ }^{3}$

Generation of critical nuclei (ref. 7)

Figure $6 a$ and $b$ show the cloud point curve (full line), $\chi_{0}$ vs. $v_{x}$ in a and temperature vs. $v_{x}$ in $b$, spinodal curve (broken line) and critical solution point (open circle) for $X$-mer / single solvent system. The calculation was carried out under the following conditions; $\mathrm{X}=3000, \mathrm{p}_{1}=0.642$ and $\mathrm{p}_{2}=0.190, \theta=307.1$ and $\psi_{0}=0.27$. These parameters were determined for polystyrene / cyclohexane system by analyzing the critical point data through use of KamideMatsuda method in 1984. ${ }^{\text {Th }}$ The area, surrounded by cloud point curve and spinodal curve is the metastable region where the critical nuclei are formed as previously mentioned.

Nuclei with a radius of $S_{C N}$ are formed by thermal fluctuation, which can be regarded as concentration fluctuation only and nucleus thus formed is considered to be in equilibrium with the region immediately surrounded as a sphere with a radius of $S_{0}$. Consider first the isothermal process. Evidently, activation energy of formation of nucleus $\Delta \phi$ is a function of $S$ and is expressed in the same form as derived for nucleation from polymer melt, ${ }^{8}$

$\Delta \phi(\mathrm{S})=\frac{4}{3} \pi \mathrm{S}^{3} \Delta \mathrm{f}_{\mathrm{v}}+4 \pi \mathrm{S}^{2} \sigma$,

where $\Delta f_{v}$ is the free energy change of coagulation per unit volume, and is defined as the

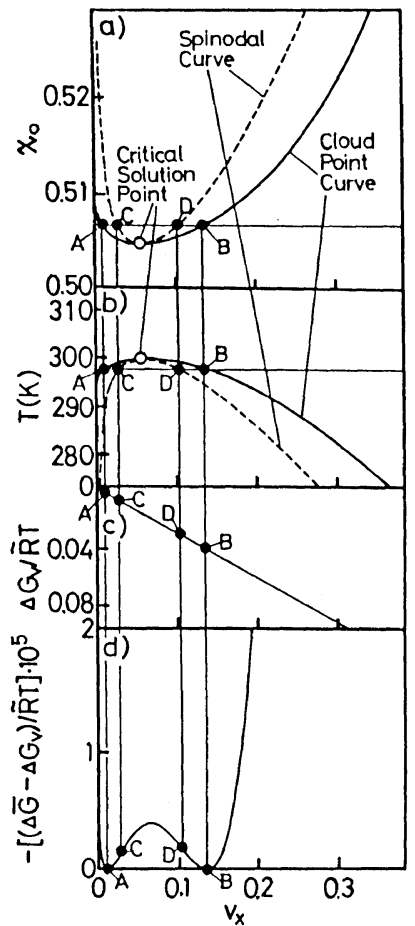

Figure 6. Cloud point curve $(a, b)$ and Gibbs free energy of mixing difference between the average Gibbs free energy of coexisting phases $A$ and $B, \Delta \bar{G}\left(v_{x}{ }^{0}\right)$ and the Gibbs free energy change of mixing per unit volume of metastable single phase, $\Delta G_{v}\left(v_{x}{ }^{0}\right)$;

$\Delta \mathrm{f}_{\mathrm{v}}=\Delta \overline{\mathrm{G}}\left(\mathrm{v}_{\mathbf{x}}{ }^{\mathbf{0}}\right)-\Delta \mathrm{G}_{\mathrm{v}}\left(\mathrm{v}_{\mathbf{x}} \mathbf{0}^{\mathbf{0}}\right)$, 
and $\sigma$ is an interfacial free energy between the nucleus and the surrounding space. The coordinates of coexisting phases $A$ and $B$ are determined first by applying chemical potentials into the Gibbs two phase equilibrium equations, respectively, and average free energy can be obtained by use of eq 18

$\Delta \bar{G}\left(v_{x^{0}}\right)=\frac{\Delta G_{v}\left(v_{X}(2)\right)-\Delta G_{v}\left(v_{X}(1)\right)}{v_{X}(2)-v_{x}(1)}\left(v_{x}{ }^{0}-v_{x(1)}\right)+\Delta G_{v}\left(v_{x}(1)\right)$,

and $\Delta f_{v}$ can be calculated. The radius of critical nuclei $S_{C N}$ is given by $S_{C_{N}}=-2 \sigma / \Delta f_{v}$. When a nucleus formed at a given instant has a radius $S_{N}$ larger than $\mathrm{S}_{\mathrm{CN}}$, the nucleus will continue to grow spontaneously. By substituting $S_{C N}$ into eq 16 , the activation energy of formation of critical nucleus $\Delta \phi_{C N}$ is written as $\Delta \phi_{C N}=(16 \pi / 3)\left(\sigma^{3} / \Delta f_{v}{ }^{2}\right)$.

Figure $6 \mathrm{c}$ and $\mathrm{d}$ show $\Delta \mathrm{G}_{\mathrm{v}}$ and the difference between $\Delta \mathrm{G}_{\mathrm{v}}$ and $\Delta \overline{\mathrm{G}}$ (i.e., $-\Delta f_{v}$ ) at the phase separation temperature $297.7 \mathrm{~K}$, which was converted from $\chi_{0}$ value. $\Delta G_{v}$ vs. $v_{x}$ curve seems roughly linear but the difference of $\Delta G_{v}$ and $\Delta \bar{G}$ has two minimums at coexisting points $A$ and $B$, and two inflection points $C$ and $D$, called spinodal points.

\section{RESULTS AND DISCUSSION}

Table I collects the value of $\Delta \mathrm{f}_{\mathrm{v}}, \sigma$ and $\mathrm{S}_{\mathrm{CN}}$ which was calculated under the condition of polystyrene $(X=300) /$ cyclohexane. $S_{C N}$ have the order of several hundred $\mathrm{nm}$ and increase as initial concentration increases. Here, interfacial free energy $\sigma$ between polymer-1ean and rich phases of polystyrene / cyclohexane solution was experimentally determined.

Table I. Size of critical nucleus of polymer / solvent system.

\begin{tabular}{llllll}
\hline $\mathbf{v}_{\mathbf{X}}{ }^{0}$ & $\mathbf{v}_{\mathbf{p}(1)}$ & $\mathbf{v}_{\mathbf{p}(2)}$ & $\Delta \mathrm{f}_{\mathrm{v}} / \mathrm{J} / \mathrm{cm}^{3}$ & $\sigma / \mathrm{J} / \mathrm{m}^{2}$ & $\mathrm{~S}_{\mathrm{CN}} / \mathrm{nm}$ \\
\hline 0.04 & 0.02 & 0.2948 & -301 & $8.07 \times 10^{-5}$ & $5.36 \times 10^{2}$ \\
0.055 & 0.04 & 0.2443 & -66.3 & $5.43 \times 10^{-5}$ & $1.64 \times 10^{3}$ \\
0.075 & 0.06 & 0.2083 & -26.4 & $3.73 \times 10^{-5}$ & $2.83 \times 10^{3}$ \\
0.090 & 0.08 & 0.1791 & -4.65 & $2.42 \times 10^{-5}$ & $1.04 \times 10^{4}$ \\
0.105 & 0.10 & 0.1542 & -0.325 & $1.30 \times 10^{-5}$ & $7.99 \times 10^{4}$ \\
0.121 & 0.12 & 0.1322 & $-6.35 \times 10^{-4}$ & $2.90 \times 10^{-6}$ & $9.14 \times 10^{6}$ \\
\hline
\end{tabular}

Figure 7 shows the interfacial free energy between air and polystyrene solution $\sigma_{0}$ measured by Wilhelmy method and plotted against polymer concentration in semi-logarithm scale. ${ }^{7}$ From the initial slope of the plots we can get the experimental equation of $\sigma_{0}$. If the interfacial free energy of the two coexisting phase $\sigma$ is assumed to be approximated by the difference of $\sigma_{0}$ which have the same concentration of $\mathrm{co}^{-}$ existing phases, we can get the equation.

$\sigma=6.9 \times 10^{-5} \log _{10} \frac{\mathrm{v}_{\mathrm{p}(2)}}{\mathrm{v}_{\mathrm{p}(1)}}$.

In the calculation of the size of

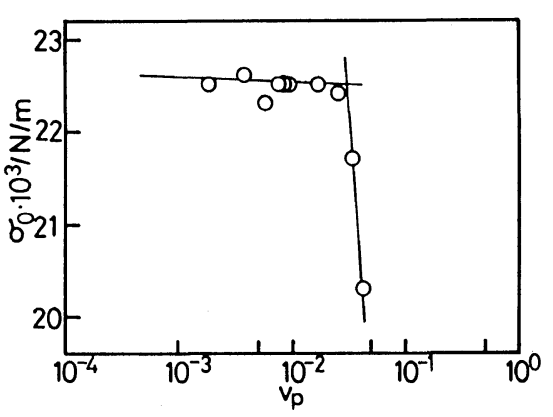

Figure 7. Surface free energy critical of polystyrene / cyclohexane. as consisting of cellulose acetate and water was employed. By use of $\sigma$ o of polystyrene / cyclohexane system $S_{C N}$ approaches to the reasonable value, which is about $1 / 2 \sim 1 / 3$ smaller than the present value.

It should be emphasized that in this case, although the phase equilibrium has not yet been attained over the whole system, phase volume ratio of polymer-lean phase to polymer-rich phase, $R$ for the 
local equilibrium region surrounding each nucleus, i.e., $R=\frac{S_{0}^{3}-S_{C N}^{3}}{S_{C N}{ }^{3}}=\frac{S_{0}\left(t_{i}\right)^{3}-S_{N}\left(t_{i}\right)^{3}}{S_{N}\left(t_{i}\right)^{3}}$,

is considered to coincide with $\mathrm{R}$ for the whole system, defined by:

$R \equiv \frac{V_{(1)}}{V_{(2)}}=\frac{V_{X(2)}-V_{X}{ }^{0}}{V_{X}{ }^{0}-V_{X}(1)}$.

Here, $V_{(1)}$ and $V_{(2)}$ are volume of polymer-lean and -rich phases, respectively, $v_{p(1)}$ and $v_{p(2)}$, the polymer volume fraction of polymerlean and polymer-rich phases, respectively and $\mathbf{v}_{\mathbf{p}}{ }^{0}$, initial polymer volume fraction.

Growth of Primary Particle by Diffusion (ref.7)

The profile of polymer concentration around a critical nucleus is demonstrated in Figure $8 \mathrm{a}$. Immediately after the generation of nucleus, the polymer molecules in the outer phase, based on the concentration difference $v_{p}{ }^{0}-v_{p}(1)$, diffuse into the sphere (hatcher area). The number of the polymer molecules diffusing through the unit area of the sphere surface from the outer phase per unit time is given by solving the general equation of diffusion.

$\frac{\partial \mathbf{v}_{\mathbf{p}}}{\partial \mathrm{t}}=\mathrm{D} \nabla^{2} \mathbf{v}_{\mathbf{p}}$

Here, $D$ is the diffusion coefficient and $t$, the growing time of nucleus by diffusion and initial condition is given as follows:

$\begin{array}{ll}\mathbf{v}_{\mathbf{p}}{ }^{0}(S, 0)=\mathbf{v}_{\mathbf{p}}(1) & \text { for } S_{N}\left(t_{i}\right)<S<S_{0}\left(t_{i}\right) \\ v_{p}{ }^{0}(S, 0)=v_{p^{0}} & \text { for } S>S_{0}\left(t_{i}\right)\end{array}$

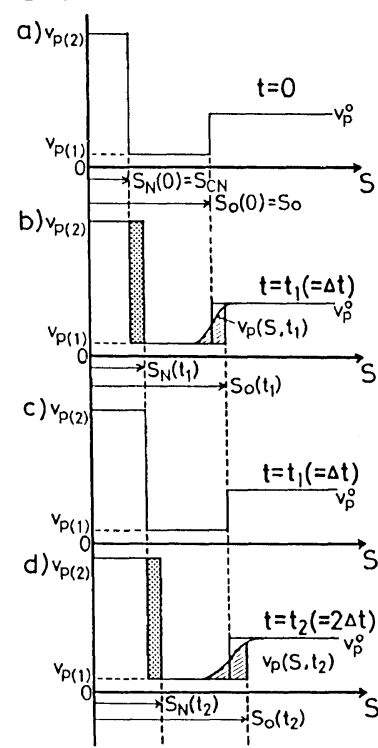

of a critical nucleus and the concentration

profile around the nucleus at $t=t_{1}$ is illustrated

in Figure $8 \mathrm{~b}$. If we can also assume that the

thermodynamic equilibrium holds even at $t=t_{1}$

Figure 8. Concentration profile of growing particle

between the nucleus and the surrounding sphere, the diffusion of polymer molecules from the outer phase into sphere will instantly result in an increase in the radius of the nucleus yielding $S_{N}\left(t_{1}\right)$ (shadowed area). This can be understood as nucleus growth. When the thermodynamic equilibrium between the nucleus and its surrounding sphere holds even at $\mathrm{t}=\mathrm{t}_{2}$ and the radius of the nucleus increases from $S_{N}\left(t_{1}\right)$ to $S_{N}\left(t_{2}\right)$. Growth rate of nucleus is given by following equation,

$\frac{\partial S_{N}(t)}{\partial t}=\frac{1}{v_{p(2)} S_{N}(t)^{2}} \int_{S_{C N}}^{S_{0}(t)}\left(\frac{\partial v_{p}(S, t)}{\partial t}\right) S^{2} d S$, where $S_{N}(t)$ is the radius of growing particle at time $t$. In deriving eq 24, mass valance equation is used.

Figure 9 presents the scheme of nucleation and growth of nuclei by diffusion of the whole system. At time $t_{1}$ some critical nuclei are generated in the system. As the nucleus grows in this manner, the surrounding polymerlean phase sphere with a radius of $S_{0}$
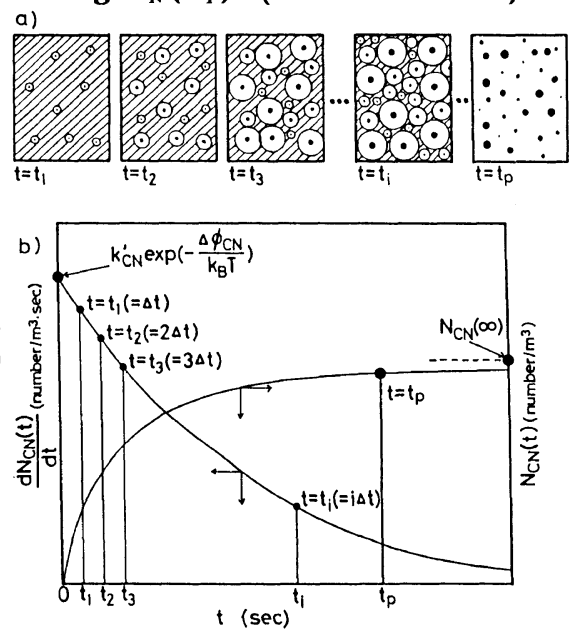

Figure 9. Scheme of nucleation and growth of particles. 
also continues to become bigger. During the growth of the nucleus, the outer homogeneous phase will disappear completely due to consumption by expansion of the polymer-lean phase sphere and generation of critical nuclei. In other words, growing polymer-rich particles with various size including critical nuclei are distributed in polymer-lean phase space at time $t_{p}$. All the nuclei at this instant are conventionally defined as primary particles. Moreover, nucleation will stop at $t_{P}$ because the phase equilibrium of total system is realized.

We assume that nucleation is always absolutely sporadic and spinodal decomposition is never predominant, then the rate of production of critical nuclei per unit volume is given by eq 25 ,

$\frac{d N_{C N}(t)}{d t}=k_{C N} \exp \left(-\frac{\Delta \phi_{C N}}{k_{B} T}\right)$,

$\left.\begin{array}{l}\text { where } \\ \mathrm{k}_{\mathrm{CN}}=\mathrm{k}^{\prime}{ }_{\mathrm{CN}}\end{array} 1-\frac{\mathrm{N}_{\mathrm{CN}}(\mathrm{t})}{\mathrm{N}_{\mathrm{CN}}(\infty)}\right\}$.

Here, $N_{C N}(t)$ and $N_{C N}(\infty)$ is the number of critical nuclei per unit volume at time $t$ and infinite time, $k^{\prime}{ }_{C N}$ is the rate constant, $k_{B}$, Boltzmann constant. The total volume of $S_{0}$ spheres including growing particles at $t, V_{0}(t)$ is expressed by eq 27 , ?

$\mathrm{V}_{0}(\mathrm{t})=\int_{0}^{\mathrm{t}} \frac{\mathrm{dN}_{\mathrm{CN}}(\tau)}{\mathrm{d} \tau} \cdot \frac{4 \pi}{3} \mathrm{~S}_{0}(\mathrm{t}-\tau)^{3} \mathrm{~d} \tau$

At the instant when the phase equilibrium is attained, $V_{0}(t)=1$ holds. In other words, in order to estimate $t_{P}$, this integral equation should be solved. Since $\mathrm{dN}_{\mathrm{CN}} / \mathrm{dt}$ can be evaluated from $\mathrm{N}_{\mathrm{CN}}(\infty), \mathrm{k}^{\prime} \mathrm{CN}, \Delta \phi_{\mathrm{CN}}$ and $\mathrm{T}, \mathrm{S}_{\mathrm{O}}$ together with $S_{N}$ can be calculated and after all $t_{P}$ can be determined. Here, $\mathrm{N}_{\mathrm{CN}}(\infty)$ should be below the number of polymer molecules per unit volume.

Figure 10 shows $\mathrm{dN}_{\mathrm{CN}}(\mathrm{t}) / \mathrm{dt}$ (in $\mathrm{a}, \mathrm{c}, \mathrm{e}$ ) and size distribution of the primary particles at $t_{P}$, $\mathrm{N}_{P P}\left(\mathrm{~S}, \mathrm{t}_{\mathrm{P}}\right.$ ) (in $\mathrm{b}, \mathrm{d}, \mathrm{f}$ ), normalized by $\mathrm{N}_{\mathrm{CN}}(\infty)$. The mean particle size shifts to smaller $S_{1}$ side with an increase in $\mathrm{N}_{\mathrm{CN}}(\infty)$. Extremely

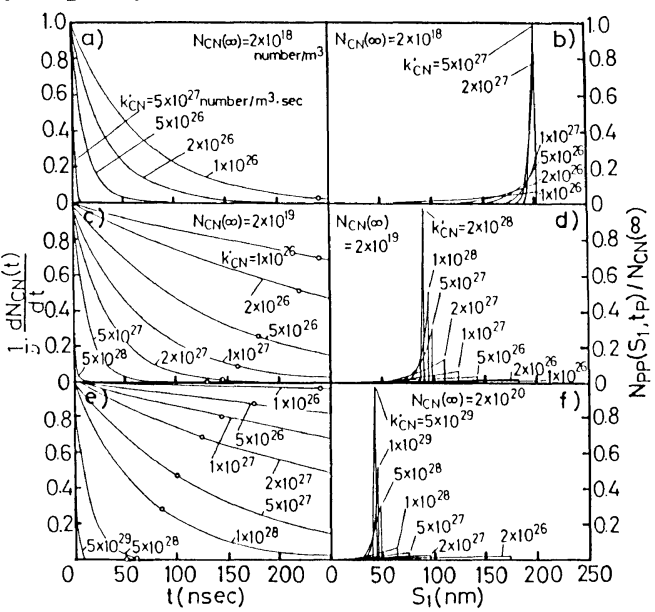

Figure 10. Nucleation rate and size distribution of primary particle.

sharp distribution of the primary particles is obtained for larger $k^{\prime}{ }_{\mathrm{CN}}$, in other words, the primary particles are uniform in size.

Growth of secondary particle by amalgamation (ref. 9)

Likewise, we can consider that there are at the initial stage two elementary steps. The nucleation and growth of nuclei by diffusion, which occur concurrently until $t=t_{p}$. We define the particles at that instant $t_{p}$ as primary particle.

The primary particles collide with each other to yield larger particles which are nominated as growing particles. The growth of the primary particle to the secondary particle can be theoretically investigated by using a particle Monte-Carlo simulation methods to describe the following three steps. This is the particle simulation approach.

In first step, the primary particles is generated at random positions in a hypothetical space. This moment is defined as $t=0$. $t$ is the time of growth of growing particle by amalgamation. Assume that the primary particle has the same radius $S_{1}$. The total number of particles 
$N_{P P}$ are determined from the phase volume ratio $R$ and $S_{1}$ written as, $N_{P P}=1 /\left\{(4 / 3) \pi S_{1}{ }^{3}(R+1)\right\}$. Here, we consider only the case where the primary particles are the polymer-rich phase.

In second step, a value for the velocity displacement of the particle is given in advance and the positions of all particles is determined randomly, after unit period of time.

In third step, the distance between the particles are measured. In the case when the distance between the center of gravity of the two arbitrarily chosen particles is less than the summation of the radius of each particle, these two particles are considered to have collided, yielding a single particle by amalgamation. ${ }^{9}$

The moving velocity of the particles can be estimated by (1) energy equi-partition law or (2) the mean square displacement of brownian movement. In case (1) the growth rate seems too large and displacement of brownian movement seems appropriate. ${ }^{9}$

The effects of the initial polymer concentration $\mathbf{v}_{\mathbf{p}}{ }^{0}$ and of the two phase volume ratio $R$ on the growth rate of particles created in phase separation process is discussed (Figure 11a). The growth particles occurs in the metastable region. The ratio of the mean radius of the growing particles $\overline{\mathrm{S}}$ to that of primary particles $S_{1}, \bar{S} / S_{1}$ for a series of solutions are evaluated at points $a_{0}, b_{0}, c_{0}$ and $d_{0}$. In this case all points are located in vicinity to the cloud point curve, corresponding to $R=100$. Inspection of Figure 1la indicates that the growth rate of the particles is larger for smaller initial polymer concentration $\mathbf{v}_{\mathbf{p}}{ }^{0}$. That is, when $R$ is the same, the particle growth rate is determined by $v_{p}(1)$ and is larger as $v_{p}(1)$ is smaller. This can be reasonably explained in the following

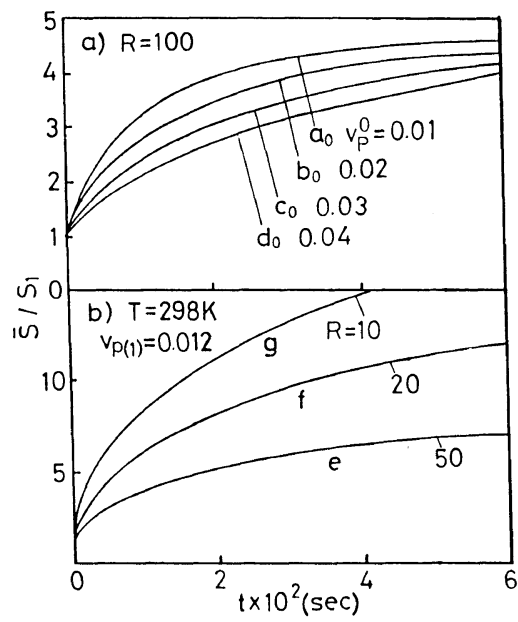

Figure 11. Average growing rate of particles. manner. The mean square displacement of the particles with the same radius is inversely proportional to viscosity of polymer-lean phase $\eta$ and the mean square displacement is larger, accordingly, the frequency of collision is larger in less viscous media. In addition, the difference in the mean particle size generated at points from $a_{0}$ to $d_{0}$ becomes less remarkable, approaching to an asymptotic value. The viscosity of the solution influences the particle growth rate, especially at the initial stage and the time necessary to attain an asymptotic value, which does not depend on $\eta$.

Figure $11 \mathrm{~b}$ shows particle growth rate of the solutions having various $R$ and constant $v_{p(1)}$. The particle grows faster for smaller $R$. When $R$ is smaller, the portion of volumes occupied by the primary particles is larger, resulting in a rapid increase of the frequency of particle-particle collision.

In the practical membrane casting process, much more complicated systems such as polymer and ternary solvents mixture system are often employed. It should be noted that for four component system the critical point is not a single point. Critical point becomes critical point curve and spinodal curve and cloud point curve become spinodal surface and cloud point surface, respectively, in the theoretical point of view. ${ }^{2}$ Figure 12 shows the critical solution point curve of cuprammonium cellulose / aq. acetone system. Here, $\overline{B C}$ is the acetone axis, $\overline{C D}$, ammonia axis, $\overline{\mathrm{DB}}$, water axis and $\overline{\mathrm{DA}}$ is the axis of cellulose plus copper. The distance of cloud point from the phase separation point can be assumed to be equal with each other. The surface enclosed by the 
full line is a deduced cloud point curve surface and the surface enclosed by the broken line is a deduced spinodal curve surface. The chain line is a critical point curve. Figure 12 shows the existence of two twophase separation regions, resulting in cosolvency. In conclusion,

Nucleation and growth of particle by diffusion were systematically studied on the basis of the thermodynamics of phase separation and the time of formation of primary particle $t_{P}$ was determined. (2) Formation of secondary particle by cellulose / aq. acetone solution. amalgamation was tried to explain by use of

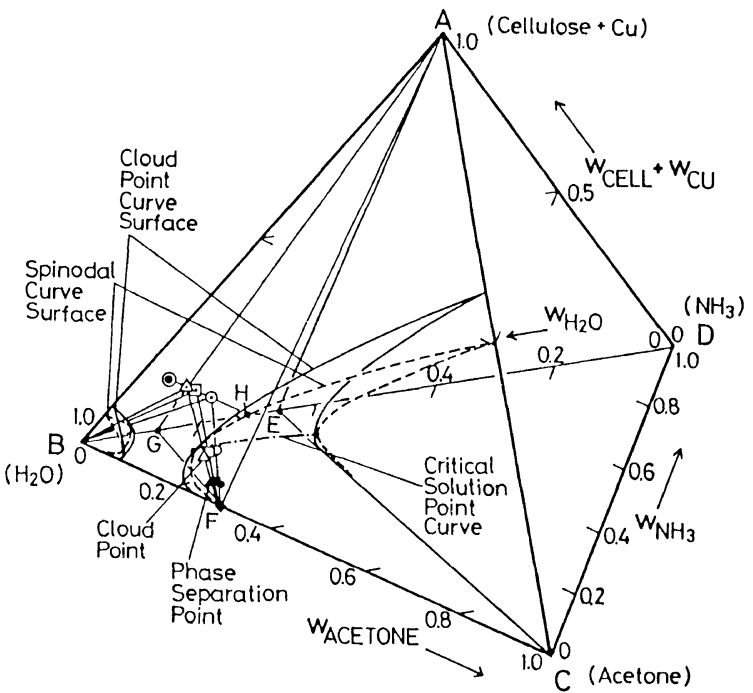

Figure 12. Phase diagram of cuprammonium cellulose / aq. acetone solution. the particle Monte-Carlo simulation approach. (3) Critical point of casting solution was determined.

The particle growth concepts involves the studies of the theoretical and computer experimental pore size distribution of the membrane using the lattice model of secondary particle, which were not presented in this paper. Summary of pore size distribution has also been published in the book previously mentioned. ${ }^{2}$

Acknowledgement

The author should like to express his most sincere gratitude to Dr. Kenji Kamide for his guidance and is grateful to thank Elsevier Science Publishers for permission to reprint Figures 1 and 4 in the book of "Polymer Science Library".

\section{REFERENCES}

1. K. Kamide and S. Manabe, in D.R. Lloyd Ed., "Materials Science of Synthetic Membranes", ACS Symposium Series, 1985, No. 269, p.197.

2. K. Kamide, "Thermodynamics of Polymer Solutions - Phase Equilibria and Critical Phenomena-", Polymer Science Library 9, A.D. Jenkins Ed., Elsevier Science Publishers, B.V., 1990.

3. K. Kamide, S. Matsuda, T. Dobashi, and M. Kaneko, Polym. J., 16, 839 (1984).

4. K. Kamide and S. Matsuda, Polym. J., 16, 825 (1984).

5. K. Kamide, S. Matsuda, and M. Saito, Polym. J., 17, 1013 (1985).

6. S. Matsuda, Polym. J., 18, 981 (1986).

7. K. Kamide, S. Matsuda, and H. Iijima, unbublished results.

8. See, for example, L. Manderkern, "Crystallization of Polymers", McGrow-Hil1 Book Co., 1964, Chapter 8.

9. K. Kamide, H. Iijima, H. Shirataki, and H. Hanahata, unpublished results. 